\title{
Characteristics of Hearing Impairment in Children Aged Six Months to Two Years with Global Developmental Delay
}

\author{
Ramesh Bhat Y, Harish Kashyap, Pushpa Kini and Shrikiran
}

Department of Paediatrics, Kasturba Medical College, Manipal, Manipal Academy of Higher Education (MAHE) University, Karnataka, India

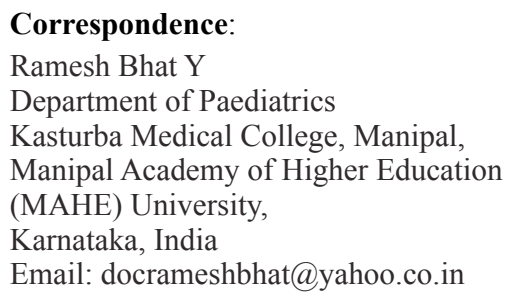

Karnataka, India

Email: docrameshbhat@yahoo.co.in

DOI: $10.3126 /$ jnps.v41i2.33961

Submitted on: $2020-12-30$

Accepted on: 2021-06-30

\section{Acknowledgements: None}

Funding: Nil

Conflict of Interest: None declared

Permission from IRB: Yes
To cite this article: Bhat RY, Kashyap H, Kini P, Shrikiran. Characteristics of Hearing Impairment in Children Aged Six Months to Two Years with Global Developmental Delay. J Nepal Paediatr Soc. $2021 ; 41(2): 140-6$.

\section{ABSTRACT}

Introduction: Children with global developmental delay (GDD) are at greater risk to have hearing impairments. These impairments interfere with developmental progress or rehabilitation effects. Hearing impairments may be correctable and if so, may improve developmental outcomes. We aimed to study the incidence, characteristics and probable risk factors of hearing impairment in children aged six months to two years with GDD.

Methods: In this prospective study, an auditory evaluation was carried out by a trained audiologist in children with GDD. Transient evoked otoacoustic emissions (TEOAE) and brainstem auditory evoked response (BERA) were assessed in these children. Hearing loss was classified based on Goodmann's classification.

Results: Of 113 children with GDD assessed, hearing impairment was identified in 35 (30.9\%) children. Of 35 children, 22 (62.8\%) had isolated sensorineural hearing loss (SNHL). Conductive hearing loss was identified in four $(11.5 \%)$ and combined hearing loss in nine $(25.7 \%)$. Of 31 children with SNHL, hearing loss was bilateral in $25(80.6 \%)$. In SNHL, hearing loss was profound in one $(3.2 \%)$, severe in $14(45 \%)$, moderately severe in three $(9.6 \%)$, and moderate in five $(16.2 \%)$. Hearing impairment was associated with $31.7 \%(20 / 63)$ children with cerebral palsy. Kernicterus was another predominant risk factor associated with SNHL. Metabolic disorders, otitis media, tuberous sclerosis, and metachromatic leukodystrophy were other conditions associated with hearing impairment.

Conclusions: Hearing impairment is accompanied by about a third of children with GDD. The hearing impairment in children with GDD tends to severe SNHL type in about $45 \%$ and bilateral in about $80 \%$.

Keywords: BERA; Children; GDD; Hearing impairment; SNHL

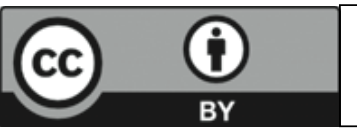

This work is licensed under creative common attribution 3.0 license 


\section{INTRODUCTION}

Significant hearing impairment, if undetected, will impede speech, language and cognitive development in children. Hearing loss in infants may be peripheral or central in origin. It may be unilateral or bilateral; conductive, sensorineural, or mixed; mild, moderate, severe or profound; of sudden or gradual onset; stable, progressive or fluctuating. The effects of hearing impairment depend on the nature and degree of the hearing loss and the individual characteristics of the child. Factors such as intelligence, medical and physical conditions including associated syndrome, family support, age at onset, time of detection, and interventions also affect the impact. About $20 \%$ of under - five children with global developmental delay (GDD) were reported to have abnormal brainstem auditory evoked response (BERA) and about $60 \%$ of children with cerebral palsy (CP) were found to have sensory neural hearing loss (SNHL).${ }^{1-3}$ Currently, the average age of detection of the significant hearing loss is reported to be 14 months. ${ }^{1}$

Developmental disabilities are estimated to affect $5 \%$ to $10 \%$ of children. ${ }^{4-6}$ The prevalence of GDD in the paediatric population is not precisely known. The estimated prevalence range from $1 \%$ to $3 \%$. GDD has variety of causes. It is associated with decreased adaptation and learning skills. Hence GDD children have problems in activities of daily living. ${ }^{6}$ It is likely that children with GDD are at higher risk to have vision and hearing impairments..$^{5-9}$ Evaluation of such impairment is usually a routine practice at the initial management of children with GDD. Hearing impairment interferes with developmental progress or rehabilitation effects. The impairments may be correctable and such correction likely positively influences developmental outcomes. Detection of a specific type of hearing deficit may also help establish the etiology of an underlying developmental disorder. ${ }^{5}$

Kwok et al studied 260 children with severe GDD and found deafness in $18 \%$ of children. ${ }^{8}$ Rupa et al. in their study involving 96 children with GDD and clinically suspected hearing loss, found hearing loss 91\%. ${ }^{9}$ Transient evoked otoacoustic emissions (TEOAE) has been found to be a screening tool for hearing impairment in children. ${ }^{5}$ In this context, we aimed to assess the incidence of hearing impairment in children aged six months to two years with GDD, study its characteristics, and to identify its risk factors.

\section{METHODS}

A prospective study was carried out over 21 months in the Department of Paediatrics, Kasturba Hospital, Manipal, India. The study population included children admitted with GDD in the age group, six months to two years. Children under six months of age or above two years of age and children whose parents were unwilling for hearing assessment were excluded. Approval from the institutional ethics committee was obtained for the study. The purpose of the study was explained to the parents and consent was obtained. Detailed history regarding developmental delay was taken and clinical examination was performed. A prestructured proforma was used to record the relevant information from individual cases. GDD was based on parental reporting measures, a checklist of developmental mile stones from Nelson Textbook of Paediatrics, and clinical examination details by two or more paediatricians. Children were subjected to auditory evaluation by a trained audiologist. TEOAE and BERA were assessed in these children. Results of TEOAE and BERA were recorded and interpreted accordingly. The workup for the etiology of GDD was carried out depending upon the findings from the history and detailed clinical examination. A duly calibrated $\mathrm{OAE}$ instrument of ILO DP Echoport 292 Usb II V.6 with foam tip was used to obtain TEOAE measurements. All the evoked potential data were obtained using Intelligent Hearing System (IHS) hardware with software version Smart EP 4.03. Hearing tests were carried out in a sound-treated air-conditioned room with adequate illumination. TEOAEs were obtained for click stimuli presented at a level of $80 \mathrm{~dB}$. Stimuli were acquired using non - linear stimulus mode where in stimuli were presented in groups of four. Signal to noise ratio above $6 \mathrm{~dB}$ at a minimum of three frequencies was considered as the presence of OAE. ABR recordings were obtained when the child was either in natural sleep or sedated with orally administered Pedicloryl (Triclofos) at a dose of $100 \mathrm{mg} / \mathrm{kg}$ body weight. The electrode site was cleaned using a skin 
Table 1. Demographic characteristics of children with GDD evaluated for hearing impairment $(n=113)$

\begin{tabular}{|lrr|}
\hline Characteristics & n & \% \\
\hline Age & & \\
$\quad$ 6 mo to 12 mo & 57 & 50.5 \\
13 mo to 18 mo & 33 & 29.2 \\
$\quad 19$ mo to 24 mo & 23 & 20.3 \\
Sex $\quad$ & \\
$\quad$ Male & 68 & 60.2 \\
$\quad$ Female & 45 & 39.8 \\
Maturity at birth & & \\
$\quad$ Preterm & 17 & 15 \\
$\quad$ Term & 96 & 85 \\
History of parental Consanguinity & 22 & 19.5 \\
\hline
\end{tabular}

preparation gel. Gold cup electrodes were placed with the help of conduction gel. The placement of electrodes was done according to the International 10-20 classification. All the evoked potential data was obtained using Intelligent Hearing System (IHS) hardware with software version Smart EP 4.03. The stimulus used was click stimulus and was presented using standard insert earphone at 40 dBnHL, EEG was acquired using a single channel (3 electrodes) with horizontal electrode montage (Fz-M1/M2). Auditory brainstem response (ABR) waveforms were analysed for the presence or absence of waves I, III and V as well as their latencies till $30 \mathrm{dBnHL}$. GDD was defined as significant delay with developmental quotient (DQ) $<70 \%$ in $\geq 2$ of the major developmental domains (Gross motor, fine motor, language and personalsocial). CP was considered when there was a disorder of posture, movement and tone due to

Table 3. Incidence, types, and laterality of hearing impairment among children with GDD

\begin{tabular}{|lrr|}
\hline & n & \% \\
\hline Hearing impairment $(\mathbf{n}=\mathbf{1 1 3})$ & & \\
Present & 35 & 30.9 \\
Absent & 78 & 69.1 \\
Type of hearing loss $(\mathbf{n}=\mathbf{3 5})$ & & \\
Sensorineural hearing Loss & 22 & 62.8 \\
Conductive hearing Loss & 4 & 11.5 \\
$\quad$ Combined hearing Loss & 9 & 25.7 \\
$\quad$ (Both SNHL+ Conductive) & & \\
Unilateral or Bilateral SNHL (n=31) & & \\
Bilateral SNHL & 25 & 80.6 \\
Unilateral SNHL & 6 & 19.4 \\
\hline
\end{tabular}

Table 2. Important physical characteristics of children with GDD $(\mathrm{n}=113)$

\begin{tabular}{lrr} 
Characteristics & n & \multicolumn{1}{c}{$\%$} \\
\hline Microcephaly & 69 & 61.1 \\
Syndromic features & 3 & 2.6 \\
Neurocutaneous markers & 11 & 9.7 \\
Dysmorphic features & 40 & 35.4
\end{tabular}

static encephalopathy acquired during brain growth in the perinatal period or early infancy. Kernicterus or sequel of bilirubin encephalopathy was defined when a neurologic syndrome resulted from a high level of unconjugated bilirubin in the neonatal period with suggestive MRI findings in the brain. Hypoxic ischaemic encephalopathy was defined as the presence of at least one of the following: not cried at birth and required resuscitation in the form of bag and mask or bag and tube ventilation; persistence of APGAR score 0 - 5 for longer than 5 min; neonatal neurologic sequelae (Seizures / coma / hypotonia) requiring care at the hospital with or without multiple organ involvement. Dysmorphic features are considered when there is a morphologic defect of an organ, part of an organ or larger region of the body resulting from an intrinsically abnormal developmental process. Microcephaly was defined as a head circumference that measures more than three standard deviations below the mean for age and sex. Metabolic disorders as an etiology for GDD was considered in the absence of significant perinatal insults, structural brain malformations, syndromic features or chromosomal abnormalities along with positive results in metabolic screening. Metabolic testing included arterial blood gas, lactate, pyruvate, ammonia levels, creatinine phosphokinase and urine analysis. No etiology for GDD was considered when there was no significant perinatal

Table 4. The severity of sensorineural hearing loss $(\mathrm{n}=31)$

\begin{tabular}{|lrr|}
\hline Severity & n & \multicolumn{1}{c|}{$\%$} \\
\hline Mild Hearing Loss & 8 & 25.8 \\
Moderate Hearing Loss & 5 & 16.2 \\
Moderately-severe Hearing Loss & 3 & 9.6 \\
Severe Hearing Loss & 14 & 45 \\
Profound Hearing loss & 1 & 3.4
\end{tabular}


Table 5. Risk factors associated with 35 GDD children with hearing impairment $(n=35)$

\begin{tabular}{lr} 
Risk Factors & n \\
\hline Cerebral palsy & 20 \\
Kernicterus & 3 \\
Metabolic Disorder & 2 \\
Tuberous Sclerosis & 1 \\
Otitis Media & 1 \\
Cerebral Atrophy & 1 \\
Arnold Chiari malformation & 1 \\
Metachromatic Leukodystrophy & 1 \\
No identifiable risk factors & 5
\end{tabular}

insult, structural brain malformations, or syndromic features and metabolic workup was negative. Hearing loss was graded based on Goodmann classification as follows: normal $<15$ dbnHL; slight hearing loss 15 - $25 \mathrm{dbnHL}$; mild hearing loss 26 - 40 dbnHL; moderate hearing loss 41-55 dbnHL; moderately severe hearing loss $56-70$ dbnHL; severe hearing loss $71-90 \mathrm{dbnHL}$ and profound hearing loss $>90 \mathrm{dbnH}$.

\section{RESULTS}

A total of 113 children with global developmental delay in the age group of six months to two years were admitted and evaluated for hearing impairments. About half of the children (50.5\%) were in the age group of six months to 12 months (Table 1). The male to female ratio was $1.5: 1$. The mean age at presentation to our centre was 13.5 months and the median was 12 months. The history of parental consanguinity was present in $19.5 \%$.

Microcephaly was found in 69 children (61.1\%) (Table2). Syndromic features were found in three $(2.6 \%)$ children. All three children were noted to Down syndrome. Dysmorphic features were found in $40(35.4 \%)$ children and they all had two or more dysmorphic features. Specific dysmorphic features found in the present study included i) Craniofacial $(n=60)$ : low set ears (Nine), brachycephaly (Six), flat occiput (Seven), squint (Seven), epicanthal folds (Six) and craniosynostosis (Seven). High arched palate and sloping forehead were observed in three children each. Malformed pinna, coarse facial features, and telecanthus were observed in two children each. Preauricular tag (One), bulbous nose (One), turricephaly (One), retrognathia (One), and synophrys (One) were the other dysmorphic features. ii) Extremities $(n=21)$ : Unilateral simian crease and clinodactyly were seen in five children each. Bilateral Simian crease, overriding of toes, and syndactyly was seen in three cases each. Polydactyly were found in two children.

An etiology was found in $64(56.6 \%)$ children with GDD. Perinatal asphyxia with hypoxic-ischemic encephalopathy (HIE) was identified as the cause in $15(13.4 \%)$ children. Structural brain malformations were found in 17 children, encephalomalacia in three and periventricular leucomalacia (PVL) in two. Cytoplasmic virus infection and metabolic disorders were found in two children each. The metabolic cause was identified in 44.8\% (22/49) GDD children. Among 113 children with GDD, 63 children were identified with CP. Spastic quadriparesis (37) and double hemiplegia (12) were the commonest types. Hypotonic CP was found in six, spastic diplegia in five and extrapyramidal $\mathrm{CP}$ in three children.

Among 113 children with GDD, hearing impairment was identified in 35 (30.9\%) children (Table 3). Of these 35 children with hearing impairment, $20(57.1 \%)$ children were males and born preterm were 5 (14.2\%). History of parental consanguinity was found in 10 (28.5\%) children. Microcephaly and dysmorphism were noted in 19 (54.2\%) and 11 (31.4\%) children respectively.

Among 35 GDD children with hearing impairment, $22(62.8 \%)$ had sensorineural hearing loss, four $(11.5 \%)$ had conductive hearing loss and nine (25.7\%) had combined hearing loss. Among four children with isolated conductive hearing loss, one child had a history of otitis media. Among 31 children with SNHL, 25 (80.6\%) had bilateral hearing loss and six (19.4\%) had unilateral hearing loss.

Among 31 children with SNHL, hearing loss was severe in $14(45 \%)$ children, moderate in five $(16.2 \%)$, moderately severe in three $(9.6 \%)$, and profound in one $(3.4 \%)$ (Table 4$)$. Nine children had both conductive and sensorineural hearing loss. 
Among 35 GDD children with hearing impairment, risk factors other than $\mathrm{CP}$ were identified in 10 children (Table 5). Among 63 children with cerebral palsy, 20 (31.7\%) had hearing impairment, nine were associated with spastic quadriplegia. The mean developmental quotient in the language domain in children with hearing loss was $38 \%$ (SD \pm 12.8 ), the range was $20-70 \%$. A hearing aid trial was given to eight children with SNHL during the study period.

\section{DISCUSSION}

The present study has identified hearing impairment in $30.9 \%$ of children with GDD. Among children with hearing impairment, hearing loss was bilateral in $80.6 \%$ and severe in $45 \%$. Because speech and language delay is often associated with GDD and could be the result of a hearing loss, hearing evaluation is mostly undertaken at the first contact. ${ }^{5-12}$ Children with GDD are at higher risk for hearing loss. ${ }^{7}$ Early detection of hearing loss at critical developmental stages can prevent or reduce subsequent adverse consequences. In the present study, half of the children $(50.5 \%)$ were in the age group of six months to 12 months. The mean age at presentation was 13.5 months. The male to female ratio was 1.5:1. In a prospective study by Meena et al. involving 200 children < two years old, $36.8 \%$ of GDD children were in the age group of six to 12 months. ${ }^{13}$ In a study by Tikaria et al. involving 100 children aged $<$ five years, the mean age was 23.6 months. $^{2}$

We noticed parental consanguinity in $19.5 \%$ study population, which is higher compared to $3 \%$ in another study. ${ }^{2}$ Microcephaly was found in $61.1 \%$ of GDD children in the present study which is much higher compared to another study which reported microcephaly in $34 \% .^{2}$ We found syndromic features in $2.6 \%$ of children which is much lower than those (14\%) mentioned by Tikaria et al. ${ }^{2}$ Dysmorphic features were found in 40 (35.4\%) children in our study. Much higher rates $(70 \%)$ of dysmorphic features were found by Tikaria et al. ${ }^{2}$

We could find etiology in $56.6 \%$ of children with GDD. Tikaria A et al. mentioned etiologic yield in
$73 \%$ and Srour et al. in $38 \%$ of the children. ${ }^{2,14} \mathrm{HIE}$ rates of $13.4 \%$ in our study is similar to those found by Tikaria et al. (15\%) and Srour et al. $(22.4 \%))^{2,14}$ Metabolic cause were identified in $44.8 \%(22 / 49)$ of GDD children in the present study. In other studies, the reported metabolic rates ranges from $2 \%$ to $4 \%{ }^{2,13,14}$ Among 113 children with GDD, 63 children were identified with CP. The higher rate of spastic CP cases in our study is similar to that reported by Singhi et al. ${ }^{15}$

The hearing impairment in GDD children varies widely. ${ }^{16-25}$ Hearing impairment was identified in $30.9 \%$ of children with GDD in the present study. Rupa et al. reported that $91 \%$ of their study children with GDD had hearing loss. ${ }^{16}$ In contrast, Tikaria et al. reported hearing loss in $20 \%$ of study children, and Kwok et al reported hearing loss in $18 \%$ among 260 children with severe global developmental delay. ${ }^{2,7}$ Among 35 GDD children with hearing impairment, $22(62.8 \%)$ had sensorineural hearing loss. Tikaria et al. found SNHL in $20 \%$ of children with GDD. ${ }^{2}$ Angulo et al. found SNHL in $18(60 \%)$ of 30 cerebral palsy children. ${ }^{3}$ Among the 31 children with SNHL in the present study, $80.6 \%$ had bilateral hearing loss and hearing loss was severe in $45 \%$ of children. In a retrospective study by Angulo et al. bilateral and unilateral SNHL were reported in $66.6 \%$ and $33.3 \%$ of children respectively. ${ }^{3}$

Among 35 GDD children with hearing impairment, risk factors included mainly CP. Among 63 children with cerebral palsy, 20 (31.7\%) had hearing impairment, nine were associated with spastic quadriplegia. Angulo et al. reported $60 \%$ of children with CP having SNHL. Hearing impairment was noted in $14 \%$ of children with CP in another Indian study. ${ }^{15}$ Other risk factors for hearing loss identified in the present study such as prematurity, kernicterus, CMV infection have been recognized earlier. ${ }^{19-24}$

We noted that the mean developmental quotient in the language domain in children with hearing loss was $38 \%$ ( $\mathrm{SD} \pm 12.8$ ). Hearing loss in infants and children may lead to lifelong deficits in speech and language acquisition. Hence, early identification and intervention for children with hearing impairment are suggested for normal children by 
researchers. ${ }^{25-30}$ Application of such strategies to GDD children is more beneficial. The American Academy of Paediatrics, the American Academy of Neurology and the British Columbia-based Treatable Intellectual Disability Endeavor (TIDE) protocols have proposed multitiered investigations of GDD. These investigations likely guide paediatricians to know the etiology and therapy optimisation. The recommendations also include formal hearing tests in these children. ${ }^{4}$ It is also stated that formal hearing testing is critical for all children with suspected GDD. The present study findings agree with these statements. Although our study is a relatively small and single centric study, we presume that our research would help shed more light in this field for the other researchers in the future.

\section{CONCLUSIONS}

Hearing impairment is associated with about $30.9 \%$ of children with GDD in young children. Isolated sensorineural hearing loss is the predominant type. Among children with SNHL, hearing loss is likely to be bilateral in the majority $(80.6 \%)$ and severe in about $45 \%$. CP and sequelae of HIE and kernicterus are the predominant risk factors associated with SNHL.

\section{REFERENCES}

1. Erenberg A, Lemons J, Sia C, Tunkel D, Ziring P. American Academy of Paediatrics, Task Force on Newborn and Infant Hearing. Newborn and infant hearing loss: detection and intervention. Paediatrics 1999;103:527-530. DOI: 10.1542/peds.103.2.527.

2. Tikaria A, Kabra M, Gupta N, Sapra S, Balakrishnan P, Gulati S, et al. Aetiology of Global Developmental Delay in Young Children: Experience from a Tertiary Care Centre in India. Natl Med J India. 2010;23(6):324-9. PMID: 21561041

3. Angulo CM, Blanco NA, Terán JG, Aledo AG, Quintela JR. Sensorineural Hearing Loss in Cerebral Palsy Patients. Acta Otorrinol aringol Esp. 2006;57(7):300-2. DOI: 10.1016/s0001-6519(06)78715-5

4. Shevell M, Ashwal S, Donley D, Flint J, Gingold M, Hirtz D, et al. Practice parameter: Evaluation of the child with global developmental delay: Report of the Quality Standards Subcommittee of the American Academy of Neurology and The Practice Committee of the Child Neurology Society. Neurology. 2003 Feb 11;60(3):367-80. DOI: 10.1212/01.wnl.0000031431.81555.16

5. Fenichel GM. Psychomotor retardation and regression. In Clinical Paediatric Neurology: a signs and symptoms approach, $4^{\text {th }}$ ed. Philadelphia: WB Saunders, 2001; 117-147.

6. Haggard M. Screening children's hearing. Br J Audiol 1992; 26: 209-215. DOI: 10.3109/03005369209076639.

7. Kwok SK, Ho PC, Chan AK, Gandhi SR, Lam DS. Ocular defects in children and adolescents with severe mental deficiency. J Intellect Disabil Res 1996; 40:330-335.DOI: 10.1111/j.1365-2788.1996.tb00638.x.

8. Shevell MI, Majnemer A, Rosenbaum P, Abrahamowicz M. Etiologic yield of subspecialists' evaluation of young children with global developmental delay. J Pediatr. 2000;136:593-8. DOI: 10.1067/mpd.2000.104817.

9. Committee on Children With Disabilities. Developmental Surveillance and Screening of Infants and Young Children. Pediatrics. 2001 Jul 1;108(1):192-6. DOI: 10.1542/peds.108.1.192.

10. Yoshinaga-Itano C, Sedey AL, Coulter DK, Mehl AL. Language of early and later-identified children with hearing loss. Pediatrics. 1998;102:1161-71. DOI: 10.1542/peds.102.5.1161.

11. Shevell M. Global Developmental Delay and Mental Retardation or Intellectual Disability: Conceptualization, Evaluation, and Etiology. Pediatr Clin North Am. 2008;55(5):1071-84. DOI: 10.1016/j.pcl.2008.07.010.

12. Battaglia A, Carey JC.Diagnostic evaluation of developmental delay/mental retardation: An overview.Am J Med Genet C Semin Med Genet. 2003 Feb 15;117(1):3-14. DOI: 10.1002/ajmg.c.10015.

13. Meenai Z, Longia S. A study on prevalence and antecedents of developmental delay among children less than 2 years attending well baby clinic. Peoples J Sci Res. 2009;2:462037.

14. Srour M, Mazer B, Shevell MI. Analysis of clinical features predicting etiologic yield in the assessment of global developmental delay. Pediatrics2006; 118: 139-45. DOI: 10.1542/peds.2005-2702. 
15. Singhi PD, Ray M, Suri G. Clinical spectrum of cerebral palsy in north India - an analysis of 1,000 cases. J Trop Pediatr. 2002;48(3):162-6. DOI: 10.1093/tropej/48.3.162.

16. Rupa V. Dilemmas in auditory assessment of developmentally retarded children using behavioural observation audiometry and brain stem evoked response audiometry. J Laryngol Otol. 1995;109:605-9. DOI: 10.1017/ s002221510013083x.

17. Behrman RE, Kleigman RM, Jenson HB, Santon BF: Nelson Textbook of Paediatrics. $20^{\text {th }}$ ed. Philadelphia, WB Saunders Company, 2016.

18. Kral A, O’Donoghue GM. Profound deafness in childhood. N Engl J Med 2010; 363:1438-50.DOI: 10.1056/ NEJMra0911225.

19. Roizen NJ. Etiology of hearing loss in children. Nongenetic causes. Pediatr Clin North Am 1999; 46:49-64. DOI: 10.1016/s0031-3955(05)70080-8.

20. Ogawa H, Suzutani T, Baba Y, Koyano S, Nozawa N, Ishibashi K et al. Etiology of severe sensorineural hearing loss in children: independent impact of congenital cytomegalovirus infection and GJB2 mutations. J Infect Dis 2007; 195:782-8. DOI: $10.1086 / 511981$.

21. Fowler KB, McCollister FP, Dahle AJ, Boppana S, Britt WJ, Passet RF. Progressive and fluctuating sensorineural hearing loss in children with asymptomatic congenital cytomegalovirus infection. J Pediatr 1997;130:624-30. DOI: 10.1016/s0022-3476(97)70248-8.

22. Tomaski SM, Grundfast KM. A stepwise approach to the diagnosis and treatment of hereditary hearing loss. PediatrClin North Am 1999; 46:35-48. DOI: 10.1016/s0031-3955(05)70079-1.

23. Cristobal R, Oghalai JS. Hearing loss in children with very low birth weight: current review of epidemiology and pathophysiology. Arch Dis Child Fetal Neonatal Ed 2008; 93:F462-8. DOI: 10.1136/adc.2007.124214.

24. deVries LS, Lary S, Dubowitz LM. Relationship of serum bilirubin levels to ototoxicity and deafness in high-risk low-birth-weight infants. Pediatrics 1985; 76:351. PMID: 4034295

25. Fortnum H, Davis A. Epidemiology of permanent childhood hearing impairment in Trent Region, 1985-1993. Br J Audiol 1997; 31:409-46. DOI: 10.3109/03005364000000037.

26. Smith RJH, Bale JF, White KR. Sensorineural hearing loss in children. Lancet. 2005;365:879-90. DOI: 10.1016/ S0140-6736(05)71047-3.

27. Robinshaw HM. Early intervention for hearing impairment: differences in the timing of communicative and linguistic development. Br J Audiol. 1995;29:315-34. DOI: 10.3109/03005369509076750.

28. Downs MP, Yoshinaga-Itano C. The efficacy of early identification and intervention for children with hearing impairment. Pediatr Clin North Am. 1999;46:79-87. DOI: 10.1016/s0031-3955(05)70082-1.

29. Joint Committee on Infant Hearing. (2007). Year 2007 position statement: Principles and guidelines for early hearing detection and intervention. Available from www.asha.org/ policy. Index terms: infants and toddlers, screening, newborns, early intervention. DOI: 10.1044/policy.PS2007-00281

30. Busa J, Harrison J, Chappell J, Yoshinaga-Itana C, Grimes A, Brookehouser PE. American Academy of Paediatrics, Joint Committee on Infant Hearing. Paediatrics. 2007;120(4):898-921. DOI: 10.1542/peds.2007-2333. 